\title{
A useful intraoperative technique for transiliac-transsacral screws: a point-to- point coaxial guide apparatus
}

\author{
Ze-hang Zheng ${ }^{\dagger}$, Fei Xu' ${ }^{\dagger}$, Zheng-qiang Luo ${ }^{*} \mathbb{D}$, Ye Ren, Tao Fu, Han-qing Xu and Bin-bin Liu
}

\begin{abstract}
Background: The transiliac-transsacral screw placement is a clinical challenge for surgeons. This study explored a point-to-point coaxial guide apparatus assisting the transiliac-transsacral screw insertion and aimed to investigate the feasibility and accuracy of the guide apparatus in the treatment of posterior ring unstable pelvic fracture compared with a free-hand technique.
\end{abstract}

Methods: A retrospective study was performed to evaluate patients treated with transiliac-transsacral screws assisted by the point-to-point coaxial guide apparatus or free-hand technique. The intraoperative data of operative time and radiation exposure times were recorded. Postoperative radiographs and $C T$ scans were performed to scrutinize the accuracy of screws position. The quality of the postoperative fracture reduction was assessed according to Matta radiology criteria. The pelvic function was assessed according to the Majeed scoring criteria at 6 months postoperatively.

Results: From July 2017 to December 2019, a total of 38 patients were included in this study, 20 from the point-to-point guide apparatus group and 18 from the free-hand group. There were no significant differences between the two groups in gender, age, injury causes, pelvic fracture type, screws level, and follow-up time $(P>0.05)$. The average operative time of the guide apparatus group for each screw was significantly less than that in the free-hand group (25.8 \pm 4.7 min vs $40.5 \pm 5.1, P$ $<0.001)$. The radiation exposure times were significantly lower in the guide apparatus group than that in the free-hand group ( $24.4 \pm 6.0$ vs $51.6 \pm 8.4, P<0.001)$. The intraosseous and juxtacortical rate of screw placement $(100 \%)$ higher than in the free-hand group (94.4\%).

Conclusion: The point-to-point coaxial guide apparatus is feasible for assisting the transiliac-transsacral screw in the treatment of posterior unstable pelvic fractures. It has the advantages of simple operation, reasonable design and no need for expensive equipment, and provides an additional surgical strategy for the insertion of the transiliac-transsacral screw.

Keywords: Pelvic fracture, Transiliac-transsacral screw, Point-to-point, Guide apparatus, Corridor

\section{Introduction}

The posterior ring unstable pelvic fracture is a common type of pelvic fracture in clinic which carries severe injuries [1]. Percutaneous iliosacral and transiliac-transsacral screws fixation provides a less invasive approach for

\footnotetext{
* Correspondence: laoaluo@aliyun.com

'Ze-hang Zheng and Fei Xu contributed equally to this work.

Department of Orthopaedics Surgery, Tongji Hospital, Tongji Medical

College, Huazhong University of Science and Technology, Wuhan 430030, China
}

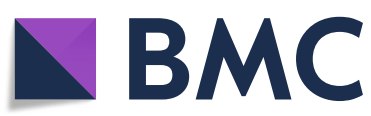

(c) The Author(s). 2021 Open Access This article is licensed under a Creative Commons Attribution 4.0 International License which permits use, sharing, adaptation, distribution and reproduction in any medium or format, as long as you give appropriate credit to the original author(s) and the source, provide a link to the Creative Commons licence, and indicate if changes were made. The images or other third party material in this article are included in the article's Creative Commons licence, unless indicated otherwise in a credit line to the material. If material is not included in the article's Creative Commons licence and your intended use is not permitted by statutory regulation or exceeds the permitted use, you will need to obtain permission directly from the copyright holder. To view a copy of this licence, visit http://creativecommons.org/licenses/by/4.0/. The Creative Commons Public Domain Dedication waiver (http://creativecommons.org/publicdomain/zero/1.0/) applies to the data made available in this article, unless otherwise stated in a credit line to the data. era loss, and lower postoperative infection rates [2, 3]. Several studies have shown that standard iliosacral screws may not provide adequate mechanical fixation, especially the pelvis with vertical instability $[4,5]$. In the case of a single screw, the transiliac-transsacral screw has a longer length than a standard iliosacral screw and provides contralateral cortical fixation, which is thought to provide improved 
resistance to vertical shear forces [6, 7]. Therefore, for patients with posterior ring unstable pelvic fracture, some surgeons, including us, prefer using transiliac-transsacral screws.

However, the transiliac-transsacral screw placement is a clinical challenge for surgeons. Its risk of extraosseous placement is theoretically increased and the operative time, the bleeding, infection, and anesthetic risk is potentially increased [2, 8]. In addition, the transiliactranssacral screw's starting point and trajectory are constrained and must be carefully planned and executed to avoid wayward screw placement [9]. Knowledge of the posterior pelvic anatomy, its variations, related imaging for sacral fixation is critical to identify the transiliactranssacral screw's safe zone and starting point [10-12]. As several previous studies mentioned and our experience, we believe the identification of the starting point and safe zone depends on improved anatomic understanding, imaging advances, preoperative screw corridor assessment, and the experience of surgeons.

Moreover, the other intraoperative difficulty of transiliac-transsacral screw insertion is to find suitable intraosseous trajectories to avoid neural compression or vascular injury and keep the trajectory and direction of the guidewire undeflected during the insertion process. The screws are inserted from the injured hemipelvis' ilium, through the sacral body and bilateral ala, and exit the contralateral iliac cortical bone, which results in a small available bone volume [2]. It means that even a slight deflection of the guidewire may cause extraosseous placement. Also, soft tissue, a wrong lateral sacral fluoroscopic intraoperative image and body position of the patient may also interfere with the procedure [8]. All these make it difficult to find the trajectory and keep the direction of the guidewire safety. Therefore, we have been exploring a solution, point-to-point coaxial guide apparatus, to guide the trajectory and direction of the guidewire, which can address this difficulty in transiliac-transsacral screw placement.

The purpose of this study was to explore the feasibility of the point-to-point coaxial guide apparatus assisting the transiliac-transsacral screw insertion and to investigate whether the guide apparatus can improve the accuracy of transiliac-transsacral screws in the treatment of posterior ring unstable pelvic fracture.

\section{Materials and methods Participants}

This study was designed as a retrospective study to evaluate patients treated with transiliac-transsacral screws assisted by the point-to-point coaxial guide apparatus or free-hand technique. Surgical placement of transiliac-transsacral screw was performed by the surgeon team in our department. The records of our hospital trauma database were retrospectively screened from July 2017 to December 2019 to identify all consecutive patients treated with posterior pelvic ring fractures. The clinical application of this technique and the retrospective review has been approved by the Ethics Committee of our university and conducted in accordance with the Helsinki Declaration of 1975 as revised in 2013. All patients have signed an informed consent form.

Inclusion criteria were (i) unstable posterior pelvic ring injuries and (ii) treatment with transiliac-transsacral screws with the technique or free-hand, and (iii) a minimum follow-up of 6 months. Patients were excluded if (i) preexisting altered skin condition and/or infection at the surgical side, (ii) treatment with transiliac-transsacral screws by other apparatuses.

\section{Outcome}

Radiological assessment included pre- and postoperative radiographs (inlet, outlet, lateral, and anteroposterior) and CT scans. All pelvic injuries were categorized into the OTA/AO classification [13]. Postoperative radiographs and CT scans were performed to scrutinize the reduction and accuracy of screws position. Its position was defined as intraosseous, juxtacortical, or extraosseous previously $[8,9]$. Complications such as nonunion, infection, nerve palsy, and paralysis were evaluated based on follow-up radiographs and retrospective chart review of the patients' medical records.

The intraoperative data of operative time and radiation exposure times were recorded. In this study, the operative time is from the incision to the safe placement of screw. The radiation exposure times refer to the number of C-arm fluoroscopy exposures to confirm the safety of the screw placement intraoperatively.

The quality of the postoperative fracture reduction was assessed according to Matta radiology criteria: excellent $(\leq 4 \mathrm{~mm})$, good $(5-10 \mathrm{~mm})$, fair $(11-20 \mathrm{~mm})$, and poor $(>21 \mathrm{~mm})$ [14]. The pelvic function was assessed according to the Majeed scoring criteria at 6 months postoperatively, which is based on the clinical findings including pain, work, sitting, sexual intercourse, and standing [15]. The score from 100 to 85 is classified as excellent, 84 to 70 as good, 69 to 55 as fair, and less than 55 as poor.

\section{Design and manufacture of the point-to-point coaxial guide apparatus}

The point-to-point coaxial guide apparatus consists of two right-angled handles that can be disassembled or combined (Fig. 1a, b). When the two handles are combined, a coaxial corridor can be formed at the distal end. The coaxial corridor can pass two cannulas, which can cannulate the guidewire of the transiliac-transsacral 

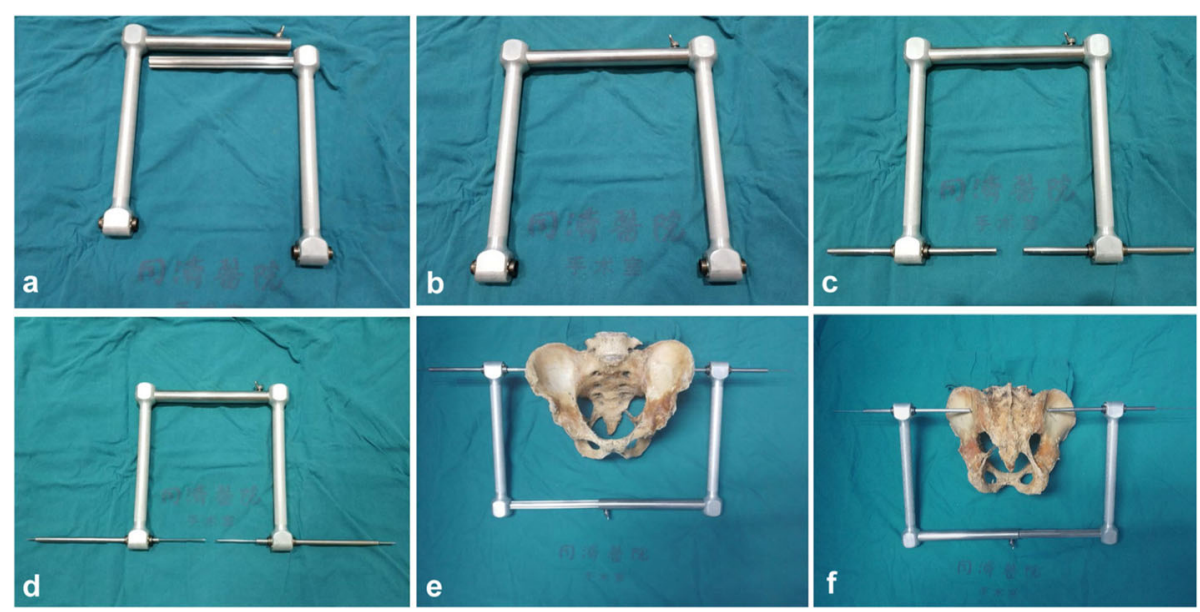

Fig. 1 The two handles are disassembled and combined $(\mathbf{a}, \mathbf{b})$. The coaxial corridor formed at the distal end can pass two cannulas (c). Insert the guide wires over the cannulas (d). As shown at the pelvis model, the two cannulas perform a point-to-point coaxial relationship by the guide apparatus and navigate the guidewire into the corridor of the $\operatorname{screw}(\mathbf{e}, \mathbf{f})$

screw (Fig. 1c). The cannulas have a serrated tip that is used to insert $2 \mathrm{~mm}$ into the ilium cortex. Since the starting point and the exiting point are guided by the two cannulas, the coaxial straight line formed by the cannula is the safety corridor of the screw (Fig. 1d). As shown at the cadaveric specimen of the pelvis, the two cannulas perform a point-to-point coaxial relationship by the guide apparatus and navigate the guidewire penetrating the corridor of the transiliac-transsacral screw from the starting point to the exiting point (Fig. 1e, f).

\section{Surgical technique}

\section{Point-to-point guide apparatus group}

An illustrative example follows with a 56-year-old man who was involved in a car accident with a complex pelvic ring injury consisting of a complete symphysis pubis disruption, fractures of superior and inferior ramus of right pubis, vertical and rotational displacement of the left hemipelvis, and left sacroiliac joint disruption (Fig. $2 \mathrm{a}-\mathrm{C}$ ). At surgery, after general anesthesia, the patient was positioned supine. The reduction of the pelvic ring is achieved by axial skeletal traction and lateral compression. Symphysis pubis disruption and fractures of superior and inferior ramus of right pubis were treated with a reconstruction plate.

First, a true lateral sacral view is the ideal starting image for the procedure to determine the safe zone and starting point of the guidewire of transiliac-transsacral screw (Fig. 2d). We inserted a threaded guide wire with $2.5-\mathrm{mm}$ diameter into the starting point through the

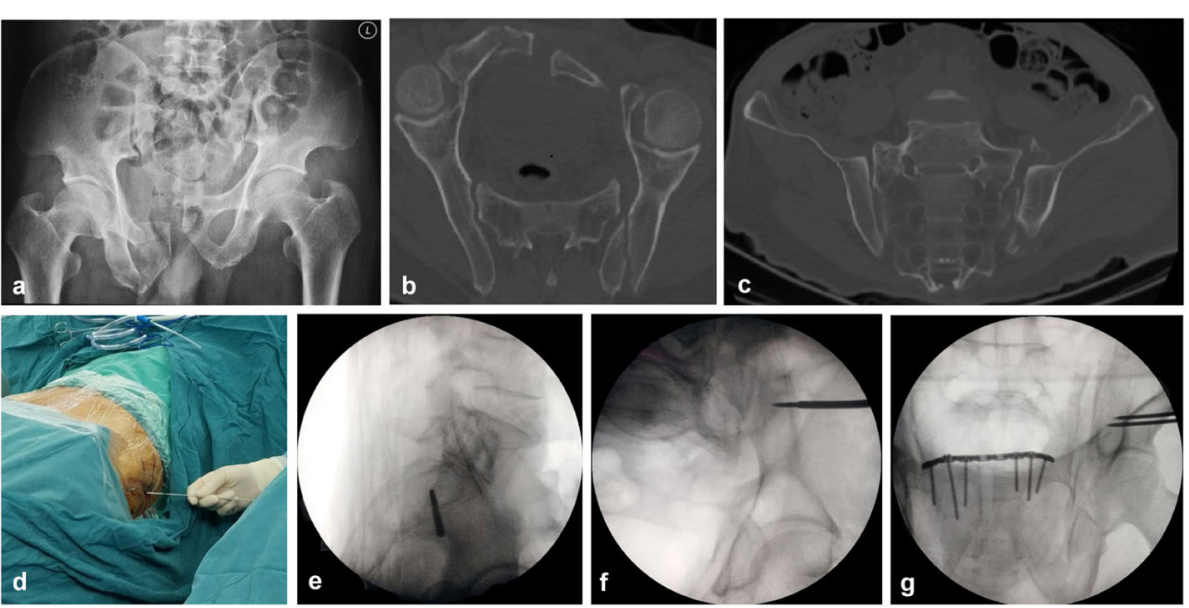

Fig. 2 Injury anteroposterior (AP) pelvis radiograph (a). Reconstruction views of axial CT (b) and coronal CT (c) showed that there was a presence of dysmorphism at the upper sacral segment, and the second sacral segment existed a corridor for a transiliac-transsacral screw. The guidewire was inserted into the starting point on the fracture side $(\mathbf{d})$ with a true lateral sacral view (e). Intraoperative fluoroscopic inlet (f) and outlet $(\mathbf{g})$ views confirmed the position of guidewire was satisfactory 
skin onto the lateral ilium on the fracture side (Fig. 2e). The inlet and outlet fluoroscopic views are used to confirm its position. Then, we inserted the cannula along the guide wire, contacted with the ilium cortex, and its serrated tip was inserted into the cortex (Fig. 2f, g). If the guidewire strayed off course, we used another guidewire for slight corrections. It deserves to be mentioned in this first step; we only pay attention to ensure the starting point of the cannula in the safety zone. Then, the other guidewire and cannula of the contralateral side were placed at the exiting point within the safe zone using the same method (Fig. $3 \mathrm{a}-\mathrm{c}$ ). At this time, the first cannula guided the starting point of the guidewire and the other cannula guided the exiting point (Fig. 3d).

Next, the point-to-point coaxial guide apparatus was installed and fixedly locked (Fig. 3e). The two cannulas formed a point-to-point coaxial relationship by the guide apparatus. Since the trajectory was directed by the two cannulas from the starting point of the safety zone to the exiting point, they formed a safe pointto-point coaxial straight transiliac-transsacral screw corridor. Subsequently, the first guidewire on the fracture side was removed and a longer guidewire was inserted along the trajectory directed by the cannulas (Fig. 3f). The longer guidewire insertion requires sequential and progressive fluoroscopic evaluation until it is just short of exiting the contralateral iliac cortex (Fig. 3g-i). Then, we removed the guide apparatus and inserted into an appropriate length transiliactranssacral screw over the guidewire. Ultimately, we ensured the position of the screw by the inlet, outlet, and lateral views again, and took an anteroposterior (AP) rollover view to assess screw length and far cortex penetration of the iliac (Fig. 4a-d). Postoperative radiographs and $\mathrm{CT}$ scans are obtained to verify reduction and implant placement (Fig. 4e-j).

\section{Free-hand group}

The operation procedure was the same as traditional transiliac-transsacral screw insertion under conventional fluoroscopy with free-hand [2].
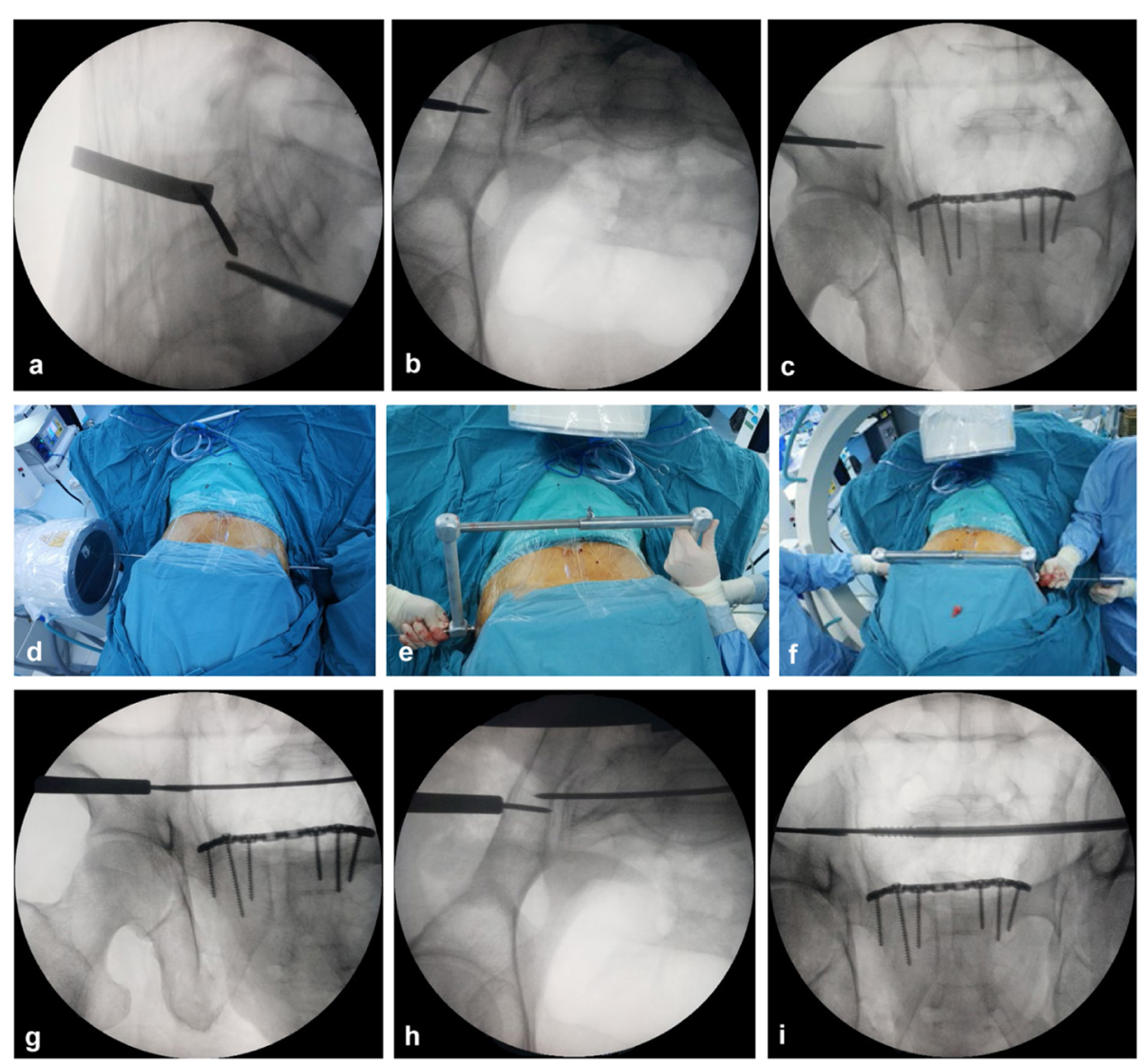

Fig. 3 Intraoperative fluoroscopic lateral (a), inlet (b), and outlet (c) views demonstrating the position of the contralateral guidewire was satisfactory. The first cannula guided the starting point of the guidewire and the other cannula guided the exiting point (d). The point-to-point coaxial guide apparatus was installed and locked (e). A longer guidewire was inserted into the screw corridor along the trajectory directed by the cannulas (f). The position of the longer guidewire can be verified by progressive fluoroscopic evaluation $(\mathbf{g}, \mathbf{h})$. When the guidewire exited the contralateral iliac cortex, we inserted into the transiliac-transsacral screw over the guidewire (i) 

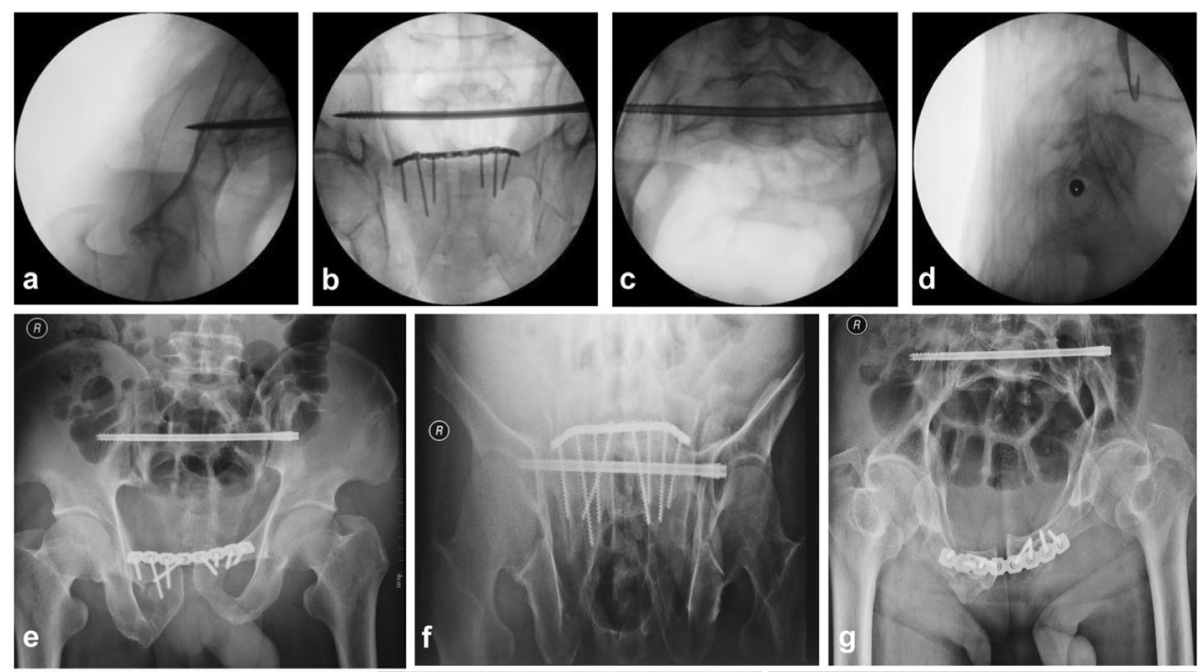

.
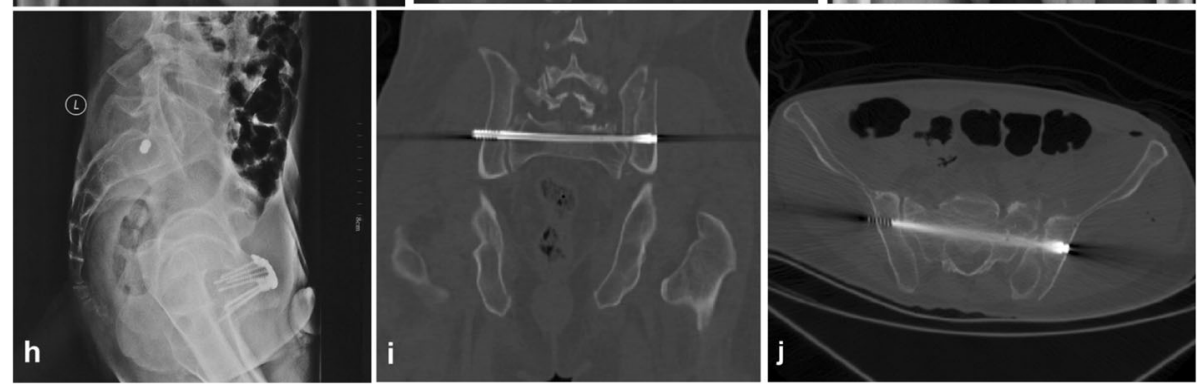

Fig. 4 Ensuring the position of the screw by the inlet, outlet and lateral views again, and taking an over-the-top view to assess screw length and far cortex penetration of the iliac (a-d). As shown in the radiographs and CT scans, the position of the transiliac-transsacral screw was satisfied except for the threads protruding through the contralateral ilium $(\mathbf{e}-\mathbf{j})$

\section{Statistical analysis}

The data were analyzed using SPSS 20.0 software (Chicago, IL, USA). The independent two-sample $t$ test was used for continuous data (presented as mean $\pm \mathrm{SD}$ ) including age, follow-up time, operation time, and radiation exposure times. The chi-square test and Fisher's exact test was used for discrete data between two groups, including gender, age distribution, injury causes, OTA/AO classification, and screws level. The Wilcoxon rank sum test was used for the data of function

Table 1 Statistic analysis of clinical indicators of the two groups

\begin{tabular}{|c|c|c|c|c|}
\hline Variable & $\operatorname{PTP}(n=20)$ & Free-hand $(n=18)$ & $T / x^{2}$ & $P$ value \\
\hline Age (years) & $47.2 \pm 17.0$ & $44.7 \pm 13.4$ & 0.483 & 0.632 \\
\hline Gender (women/men) & $6 / 14$ & $7 / 11$ & 0.333 & 0.734 \\
\hline Follow-up in months & $14.7 \pm 2.2$ & $13.8 \pm 2.1$ & 1.187 & 0.243 \\
\hline \multicolumn{5}{|l|}{ Cause of injury } \\
\hline Fall from height/ traffic accident & $3 / 17$ & $4 / 14$ & 0.329 & 0.687 \\
\hline \multicolumn{5}{|c|}{ Pelvic fracture type (OTA/AO classification) } \\
\hline Type B/ type C & $9 / 11$ & $12 / 6$ & 1.799 & 0.210 \\
\hline \multicolumn{5}{|l|}{ Screws level $(6.5 \mathrm{~mm})$} \\
\hline S1 level/S2 level & $9 / 11$ & $11 / 7$ & 0.986 & 0.352 \\
\hline \multicolumn{5}{|l|}{ Intraoperative data (mean \pm SD) } \\
\hline Operation time (min) & $25.8 \pm 4.7$ & $40.5 \pm 5.1$ & -9.160 & $<0.001^{\mathrm{a}}$ \\
\hline Radiation exposure times $(\mathrm{N})$ & $24.4 \pm 6.0$ & $51.6 \pm 8.4$ & -11.489 & $<0.001^{\mathrm{a}}$ \\
\hline
\end{tabular}

PTP point to point guide apparatus

${ }^{\text {a }}$ Statistical significance 
outcomes and quality of reduction. $P$ value less than 0.05 was considered as statistically significant.

\section{Results}

From July 2017 to December 2019, a total of 38 patients diagnosed with unstable posterior pelvic ring injuries were included in this study. There were no significant differences between the two groups in gender, age, injury causes, pelvic fracture type, and screws level $(P>0.05)$ (Table 1). From the date of surgery, all patients were required to visit our specialist clinic at 1, 2, 3, 6, 12, and 18 months postoperatively. Some patients may lose to follow-up because of multiple reasons. However, no significant differences were found between the two groups $(14.7 \pm 2.2$ months of follow-up in the guide apparatus group vs $13.8 \pm 2.1$ months in the free-hand group, $P>$ 0.05 ) (Table 1). All screws were not routinely removed.

The patients with anterior pelvic ring fractures were treated with reconstruction plates, anterior column screws, or external fixation devices. The average operative time of the point-to-point guide apparatus group for each screw was significantly less than that in the freehand group ( $25.8 \pm 4.7 \mathrm{~min}$ vs $40.5 \pm 5.1, P<0.001)$. The radiation exposure times were significantly lower in the point-to-point guide apparatus group than that in the free-hand group $(24.4 \pm 6.0$ vs $51.6 \pm 8.4, P<0.001)$ (Table 2).

A total of 38 transiliac-transsacral screws $(6.5 \mathrm{~mm}$ cannulated screw) were placed in 38 patients: 20 screws (9 S1, 11 S2) in point to point guide apparatus group and 18 screws (11 S1, 7 S2) in free-hand group. The intraosseous and juxtacortical rate of screw placement in point-to-point guide apparatus group was $100 \%$ and higher than $94.4 \%$ in the free-hand group. No complications including nerve palsy and revisions were noted postoperatively. According to Majeed scores at 6 months and Matta radiology criteria, function outcomes and quality of reduction has no significant differences between the two groups. $\left(\chi^{2}=-0.711, P=0.477 ; \chi^{2}=-\right.$ $0.107, P=0.914)$ (Table 2).

\section{Discussion}

Several studies have described the relationship of the iliac cortical density (ICD), that is, the dense area of anterior ilium located directly lateral to the sacroiliac joint, and the sacral alar anterior cortical anatomy. This relationship is paramount for identifying the safe zone and an appropriate starting point [16]. On the true lateral sacral fluoroscopic image, the ICD is coplanar with the anterior sacral alar cortical bone in the nondysmorphic sacrum. But in patients with sacral dysmorphism, like a more acute alar slope, would have less alar bone available for screw insertion [17]. This oblique dysmorphic alar osteology makes transiliac-transsacral screw fixation impossible [12, 16]. However, patients with a dysmorphic sacrum always have a safe zone at the second sacral segment that can insert a transiliac-transsacral screw [11].

The so-called safe zone, most restricted between the sacral ala and sacral neural tunnel, is the area within the sacrum where completely intraosseous transiliactranssacral screws are ideally inserted $[16,18]$. Since the pelvis can be considered as a structure of bilateral symmetry and there are two safe zones for transiliactranssacral screw at both sides of ilium [19], we regard the transiliac-transsacral screw corridor as a point-topoint (safe zone to safe zone) coaxial trajectory from the starting point of the guidewire to the exiting point. The two points are included in the safe zone of the transiliac-transsacral screw estimated by a true sacral lateral view. On the true lateral view, two points ideally will overlap to form one point. Since two points form a coaxial straight line, the exiting point, representing the starting point at the contralateral side, can be used to

Table 2 Postoperative clinical indicators of the two groups

\begin{tabular}{|c|c|c|c|}
\hline Variable & PTP $(n=20)$ & Free-hand $(n=18)$ & Range or percent \\
\hline Function outcomes (Majeed scores, $N$ ) & & & $x^{2}=-0.711, P=0.477$ \\
\hline Excellent/good & $12 / 7$ & $9 / 7$ & $95 \%$ vs $88.9 \%$ \\
\hline Fair & 1 & 2 & $5 \%$ vs $11.1 \%$ \\
\hline Quality of reduction (Matta radiology criteria, $N$ ) & & & $X 2=-0.107, P=0.914$ \\
\hline Excellent/good & $14 / 4$ & $12 / 5$ & $90 \%$ vs $94 \%$ \\
\hline Fair & 2 & 1 & $10 \%$ vs $6 \%$ \\
\hline \multicolumn{4}{|l|}{ Screw position } \\
\hline Intraosseous & 16 & 9 & $80 \%$ vs $50 \%$ \\
\hline Juxtacortical & 4 & 8 & $20 \%$ vs $44.4 \%$ \\
\hline Extraosseous & 0 & 1 & $0 \%$ vs $5.6 \%$ \\
\hline Complications & No & No & \\
\hline
\end{tabular}


find and guide the trajectory and direction of the guidewire of the transiliac-transsacral screw. This is the core principle and the most important safety aspect of the point-to-point coaxial guide apparatus.

It has been reported that 3D fluoroscopic navigation enabled safer iliosacral screw placement [20]. However, Takao et al. found that the risk factors for inaccurate positioning of iliosacral screws inserted using 3D fluoroscopic navigation were the screw insertion angle in the axial plane and the use of transsacral screws because the navigation system guides the sleeve device for guidewire insertion, not the guidewire or the screw itself; and the guidewire flexibility is also one possible reason [21]. In our study, the screw insertion angle was not a risk factor for inaccurate screw insertion as the transsacral trajectory was guided by the guide apparatus according the principle mentioned above. Moreover, although the guidewire has flexibility, the corridor of the guidewire formed by the guide apparatus is not affected by the elasticity of the skin and soft tissue around the cannulas, even in patients with truncal obesity, which allow the surgeon to keep the direction of the guidewire during inserting.

Medical robotic systems for percutaneous iliosacral screw placement have been developed [22]. Wang et al. reported that the accuracy of the robot-assisted technique was superior to the conventional free-hand technique [23]. But, using a robot-assisted navigation system adds to the cost of the surgery and many hospitals may have difficulty paying for robots [23], and there were no reports about the accuracy of the robot-assisted technique for transiliac-transsacral screw placement in the treatment of posterior ring unstable pelvic fracture.

In our study, accuracy of the point-to-point coaxial guide apparatus was superior to that of the free-hand technique. Our clinical experience with the described screw placement technique resulted in the intraosseous and juxtacortical rate of screw placement (100\%) higher than in the free-hand group (94.4\%). Also, the time required to place each screw and the radiation exposure times in the guide apparatus group was significantly better than in the free-hand group, and no complications were noted at the final follow-up. Although much time was spent in finding the exiting point, the duration of the operation and the number of fluoroscopy exposures were significantly reduced, and there are no expensive surgical instruments required because the manufacture of the guide apparatus is simpler and cheaper than a robot, CT machine, or 3D navigation [24]. Ultimately, surgeons who are adept at inserting iliosacral and transiliac-transsacral screws can master this technique with a small learning curve.

However, some limitations are inherent in this study. First, although the learning curve of this technique is small, it still requires surgeons to have rich surgical experience and correctly interpret intraoperative imaging to verify reduction and implant placement. Meanwhile, the fracture or dislocation of the sacroiliac joint may make the standard lateral view difficult to achieve; this could make it hard for us to find the exact starting point and exiting point of the guide wire. So, an accurate sacroiliac joint reduction is extremely important for this technique and it must already be achieved. Finally, this was a retrospective evaluation of our experience; a prospective study and a more sufficient sample size might give further insights in the limitations and complications of the guide apparatus.

\section{Conclusion}

The point-to-point coaxial guide apparatus is feasible for assisting the transiliac-transsacral screw in the treatment of posterior unstable pelvic fractures. Accuracy of the point-to-point coaxial guide apparatus was superior to that of the free-hand technique. It has the advantages of simple operation, reasonable design, and no need for expensive equipment, and provides an additional surgical strategy for the insertion of the transiliac-transsacral screw.

\section{Acknowledgements \\ None.}

Authors' contributions

Ze-hang Zheng and Fei Xu recorded the data, drafted the manuscript, and contributed equally to this work. Ye Ren, Tao Fu, Han-qing Xu and Bin-bin Liu analyzed and interpreted the patient data and accomplished the statistical analyses. Zheng-qiang Luo made substantial contributions in the conception of the study and supervised the conduction of it. All authors read and approved the final manuscript.

\section{Funding}

There is no funding source.

Availability of data and materials

All data analyzed during this study are included in this published article and its supplements.

Consent for publication

Not applicable.

Competing interests

The authors declare that they have no competing interests.

Received: 27 October 2020 Accepted: 17 January 2021

Published online: 28 January 2021

References

1. Sathy AK, Starr AJ, Smith WR, Elliott A, Agudelo J, Reinert CM, et al. The effect of pelvic fracture on mortality after trauma: an analysis of 63,000 trauma patients. J Bone Joint Surg Am. 2009;91:2803-10.

2. Gardner MJ, Routt ML Jr. Transiliac-transsacral screws for posterior pelvic stabilization. J Orthop Trauma. 2011;25:378-84.

3. Bousbaa H, Ouahidi M, Louaste J, Bennani M, Cherrad T, Jezzari H, et al. Percutaneous iliosacral screw fixation in unstable pelvic fractures. Pan Afr Med J. 2017;27:244.

4. Sagi HC, Ordway NR, DiPasquale T. Biomechanical analysis of fixation for vertically unstable sacroiliac dislocations with iliosacral screws and symphyseal plating. J Orthop Trauma. 2004;18:138-43. 
5. Mears SC, Sutter EG, Wall SJ, Rose DM, Belkoff SM. Biomechanical comparison of three methods of sacral fracture fixation in osteoporotic bone. Spine (Phila Pa 1976). 2010;35:E392-5.

6. Zhao Y, Li J, Wang D, Liu Y, Tan J, Zhang S. Comparison of stability of two kinds of sacro-iliac screws in the fixation of bilateral sacral fractures in a finite element model. Injury. 2012;43:490-4.

7. Zhao Y, Zhang S, Sun T, Wang D, Lian W, Tan J, et al. Mechanical comparison between lengthened and short sacroiliac screws in sacral fracture fixation: a finite element analysis. Orthop Traumatol-Sur. 2013;99: 601-6.

8. Eastman JG, Kuehn RJ, Chip Routt ML Jr. Useful Intraoperative Technique for Percutaneous Stabilization of Bilateral Posterior Pelvic Ring Injuries. J Orthop Trauma. 2018;32:e191-e7.

9. Lucas JF, Routt ML Jr, Eastman JG. A useful preoperative planning technique for transiliac-transsacral screws. J Orthop Trauma. 2017:31:e25-31.

10. Ziran BH, Wasan AD, Marks DM, Olson SA, Chapman MW. Fluoroscopic imaging guides of the posterior pelvis pertaining to iliosacral screw placement. J Trauma. 2007;62:347-56 discussion 56

11. Mendel T, Noser H, Kuervers J, Goehre F, Hofmann GO, Radetzki F. The influence of sacral morphology on the existence of secure $\mathrm{S} 1$ and $\mathrm{S} 2$ transverse bone corridors for iliosacroiliac screw fixation. Injury. 2013;44: 1773-9.

12. Konig MA, Sundaram RO, Saville P, Jehan S, Boszczyk BM. Anatomical considerations for percutaneous trans ilio-sacroiliac S1 and S2 screw placement. Eur Spine J. 2016;25:1800-5.

13. Marsh JL, Slongo TF, Agel J, Broderick JS, Creevey W, DeCoster TA, et al. Fracture and dislocation classification compendium - 2007: Orthopaedic Trauma Association classification, database and outcomes committee. J Orthop Trauma. 2007;21:S1-133.

14. Tornetta P 3rd, Matta JM. Outcome of operatively treated unstable posterior pelvic ring disruptions. Clin Orthop Relat Res. 1996;329:186-93.

15. Majeed SA. Grading the outcome of pelvic fractures. J Bone Joint Surg Br. 1989;71:304-6

16. Miller AN, Routt ML Jr. Variations in sacral morphology and implications for iliosacral screw fixation. J Am Acad Orthop Surg. 2012;20:8-16.

17. Giannoudis PV, Papadokostakis G, Alpantaki K, Kontakis G, Chalidis B. Is the lateral sacral fluoroscopic view essential for accurate percutaneous sacroiliac screw insertion? An experimental study. Injury-Int J Care Injured. 2008;39: 875-80.

18. Mendel T, Noser H, Wohlrab D, Stock K, Radetzki F. The lateral sacral triangle-A decision support for secure transverse sacroiliac screw insertion. Injury-Int J Care Injured. 2011;42:1164-70.

19. Lee JJ, Rosenbaum SL, Martusiewicz A, Holcombe SA, Wang SC, Goulet JA. Transsacral screw safe zone size by sacral segmentation variations. J Orthop Res. 2015;33:277-82.

20. Matityahu A, Kahler D, Krettek C, Stockle U, Grutzner PA, Messmer P, et al. Three-dimensional navigation is more accurate than two-dimensional navigation or conventional fluoroscopy for percutaneous sacroiliac screw. fixation in the dysmorphic sacrum: a randomized multicenter study. J Orthop Trauma. 2014;28:707-10.

21. Takao M, Hamada H, Sakai T, Sugano N. Factors influencing the accuracy of iliosacral screw insertion using 3D fluoroscopic navigation. Arch Orthop Trauma Surg. 2019;139:189-95.

22. Liu HS, Duan SJ, Liu SD, Jia FS, Zhu LM, Liu MC. Robot-assisted percutaneous screw placement combined with pelvic internal fixator for minimally invasive treatment of unstable pelvic ring fractures. Int J Med Robot. 2018;14:e1927.

23. Wang JQ, Wang Y, Feng Y, Han W, Su YG, Liu WY, et al. Percutaneous sacroiliac screw placement: a prospective randomized comparison of robotassisted navigation procedures with a conventional technique. Chin Med J (Engl). 2017;130:2527-34

24. Ghisla S, Napoli F, Lehoczky G, Delcogliano M, Habib N, Arigoni M, et al. Posterior pelvic ring fractures: Intraoperative 3D-CT guided navigation for accurate positioning of sacro-iliac screws. Orthop Traumatol Surg Res. 2018; 104:1063-7.

\section{Publisher's Note}

Springer Nature remains neutral with regard to jurisdictional claims in published maps and institutional affiliations.

Ready to submit your research? Choose BMC and benefit from:

- fast, convenient online submission

- thorough peer review by experienced researchers in your field

- rapid publication on acceptance

- support for research data, including large and complex data types

- gold Open Access which fosters wider collaboration and increased citations

- maximum visibility for your research: over $100 \mathrm{M}$ website views per year

At BMC, research is always in progress.

Learn more biomedcentral.com/submissions 